\title{
An Investigation into Flow and Heat Transfer of an Ultrasonic Micro-blower Device for Electronics Cooling Applications
}

\author{
Omidreza Ghaffari
}

Mechanical Engineering Department, Ozyegin University, Nisantepe Mah., Orman Sok Istanbul, 34794, Turkey

Stephen A. Solovitz (corresponding author)

School of Engineering and Computer Science, Washington State University Vancouver, Vancouver, WA 98686, USA

Email: stevesol@vancouver.wsu.edu

Muhammad Ikhlaq

Mechanical Engineering Department, Ozyegin University, Nisantepe Mah., Orman Sok Istanbul, 34794, Turkey

Mehmet Arik

Mechanical Engineering Department, Ozyegin University, Nisantepe Mah., Orman Sok Istanbul, 34794, Turkey

\begin{abstract}
As compact electronics increase in functionality, new electronics cooling approaches must be more effective, and they must be lower in form factor. In this paper, we investigated the cooling performance of a miniature ultrasonic micro-blower impinging upon a vertical heater. We studied the temperature response at different operating conditions, determining the optimal thermal conditions. We further examined the local flow field using the particle image velocimetry (PIV) technique at the same operating conditions, providing explanations for the heat transfer response in terms of the fluid dynamics. Heat transfer measurements show that the maximum cooling performance occurs at a jet-to-surface spacing ratio of $15<H / D<30$, and the performance slowly decays when the jet is located further away. The preferred operating frequency of the piezoelectric cooling device occurs at an ultrasonic frequency of
\end{abstract}


over $20 \mathrm{kHz}$, meaning that this device can function outside the human hearing range. The PIV results demonstrate that the jet profile in the near field deviates significantly from a traditional turbulent free jet. In the far field, it nearly matches the self-similar, fully-developed jet profile. The jet cooling performance is sensitive to the frequency, with the thermal performance dropping by a factor of six when varying by less than $1 \mathrm{kHz}$ from the peak. At the optimal heat transfer condition, the coefficient of performance is measured at approximately three, which is lower than that of some synthetic jets.

Keywords: Ultrasonic blower, Impingement, Electronics cooling, Particle image velocimetry, Synthetic jet, Thermal management. 


\section{Introduction}

Modern electronics have been decreasing in size for decades, so their cooling systems must continually improve in efficiency at reduced thermal estates too. In particular, compactness is vital, which is challenging, because typical thermal management uses relatively large fans and heat sinks. Powerful cooling techniques such as, advanced liquid cooling, additional coolant and structures are required, somewhat counteracting the improvement with liquid heat transfer. Ideally, thermal management solution should be economical, low volume, and localized on the powered devices. Fortunately, some recent advances in synthetic jet devices $[1,2]$ may provide a potential solution in both air and liquids cooling.

Synthetic jets use an oscillating structure near an orifice, which produces a periodic jet outflow and sink inflow. When averaging over time, this leads to an axial jet, which can be directed towards a powered device. Unlike traditional impingement cooling, the jet is supplied by ambient fluid, as opposed to an additional coolant. Newer actuators also have more compact structures [2], potentially reducing volume further.

Over the past 30 years, a number of researchers have examined the thermal performance of synthetic jet and similar micro-flow devices. Early studies with pulsating jets increased convection by a factor of four [3,4], while later piezoelectric devices produced similar performance in a smaller planform [5]. Improved actuator structures led to higher jet speeds, leading to heat transfer enhancement by 10 to 15 times [2, 6, 7]. Most synthetic jets have used circular exits, while a recent design by Ghaffari et al. [2] applied a slot jet with an aspect ratio of 8:1 and a rectangular cross section. In addition, the driving piezoelectric disk was aligned in-line with the jet axis, resulting in high speeds while remaining at a compact size.

One of the prime limitations of synthetic jets is their high noise output. Piezoelectric designs generate rapid diaphragm motion, producing a high velocity jet stream that mixes with the ambient air. Unfortunately, effective synthetic jets often require operation at the actuator resonant frequency to maximize jet exit velocity, coinciding with the highest noise output [810]. For example, Arik [11] observed that a synthetic jet with a 1-mm orifice achieved its peak jet velocity of $90 \mathrm{~m} / \mathrm{s}$ at a resonant frequency of $3.6 \mathrm{kHz}$. However, the corresponding 
acoustic intensity was $73 \mathrm{dBA}$, comparable to loud appliances like vacuum cleaners and leaf blowers.

There are some existing methods that can decrease the noise level of synthetic jets. First, designers may use slightly off-resonant actuation conditions to produce a lower noise, but this also reduces the heat transfer [12]. Second, a new actuator topology can be developed to reduce noise generation without decreasing the peak jet velocity. Jabbal and Kykkotis [13] designed a synthetic jet with two chambers and a corrugated-lobed orifice, which had a $20 \mathrm{~dB}$ (or $32 \%$ ) noise reduction compared to a single chamber, circular orifice device at the Helmholtz frequency. This new device only had a $7 \%$ reduction in peak jet velocity, while maintaining the same discharge area. However, it required a more complex structure that may be challenging to miniaturize further.

Another intriguing solution is to operate the jet at a frequency higher than the audible level for humans, which is approximately $20 \mathrm{kHz}$. Figure 1 shows an image of one such ultrasonic piezoelectric micro-blower, manufactured by Murata Manufacturing Co., Ltd. [14]. This device uses two cavities, which permits inlet and exhaust flows through orifices of differing sizes and locations.

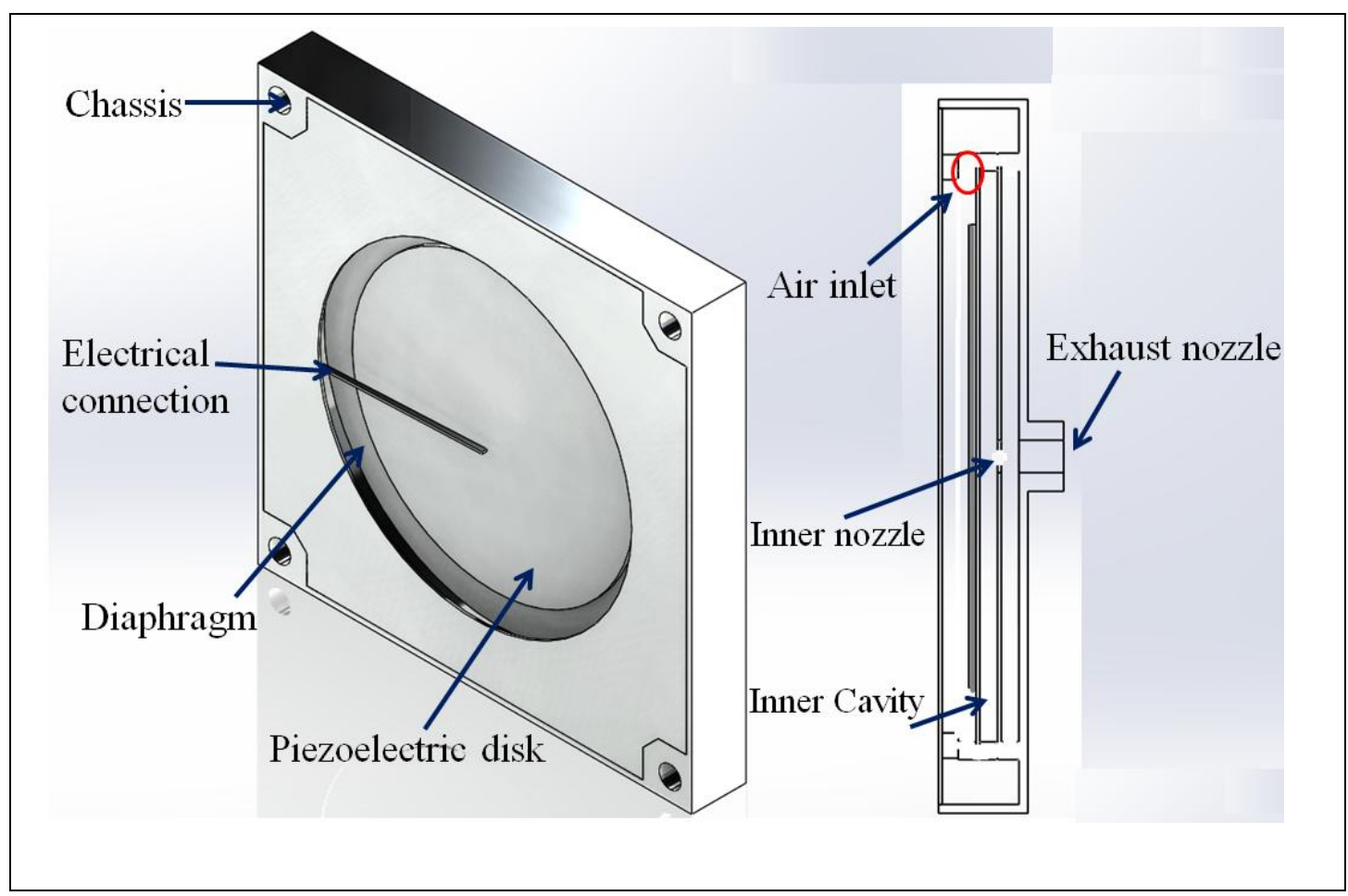


Figure 1. Schematic of the piezoelectric ultrasonic micro-blower (Footprint area of $20 \mathrm{~mm} \mathrm{x}$ $20 \mathrm{~mm}$; thickness of $2 \mathrm{~mm}$; two millimeter-scale ports to the inner cavity for inflow and exhaust).

Figure 2 shows how the jet flow is generated. As with many synthetic jets, the primary driver is a piezoelectric disk, which is located on one surface of the inner cavity. An alternating current is applied in a sinusoidal waveform to the disk, which expands and contracts in turn. During the motion, this disk pushes an attached diaphragm, changing the volume of the inner cavity. This pulls fluid into and out of an orifice along the centerline of this cavity. Unlike with most synthetic jets, the inflow to the jet is primarily from the back of the device, where it enters the outer cavity along its circumferential perimeter. In the disk contraction phase, shown in Figure 2a, the diaphragm is pulled back, expanding the inner cavity and sucking air into it through its orifice. In the expansion phase, shown in Figure $2 b$, the diaphragm moves to contract the inner cavity, ejecting air through the orifice and the exhaust nozzle along its centerline. This process continuously repeats at the frequency of the piezoelectric vibration. The combination of alternating jet exhaust and sink inflow generates an axially-outward flow. Additional information about the device design is provided by the manufacturer [14]. The dual-cavity design is unique, allowing air inflow from a different location than the exhaust nozzle. In contrast, most traditional synthetic jets use the same orifice for inflow and outflow.

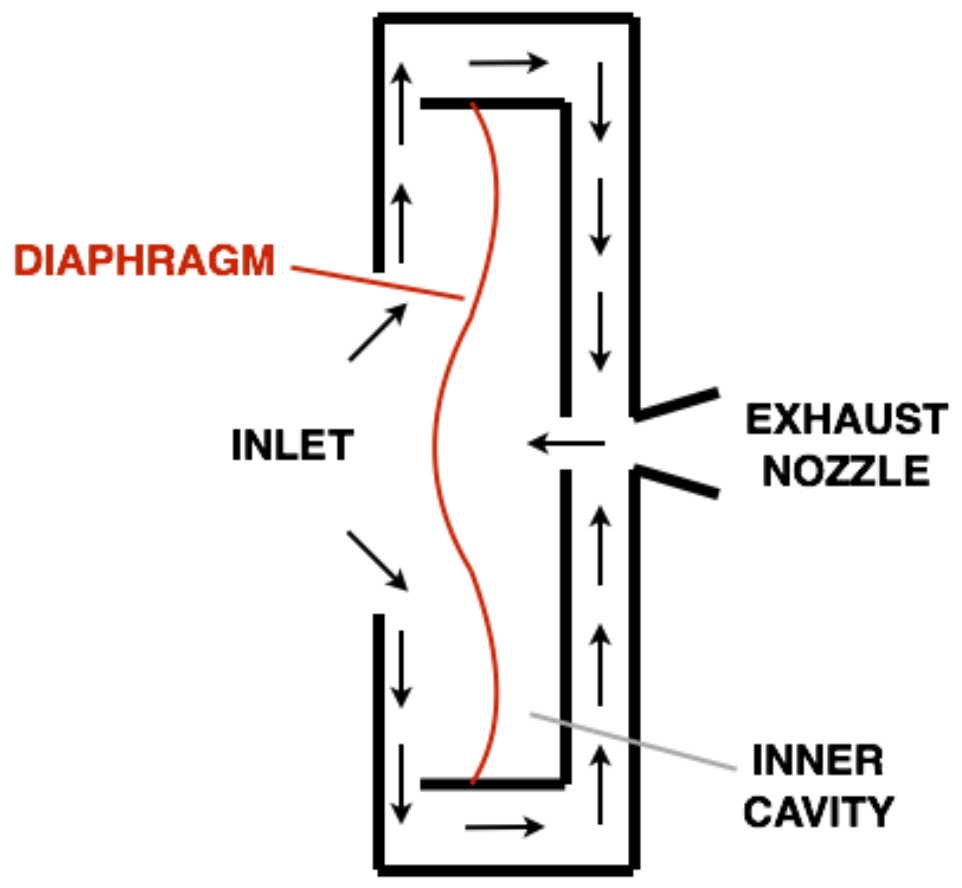

(a)

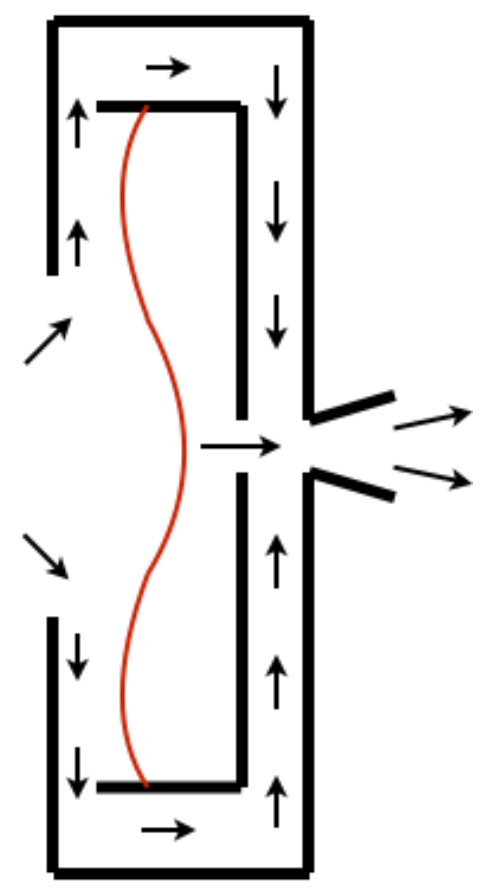

(b) 
Figure 2. Schematic of micro-blower operation in a) Suction b) Ejection. The images are not to scale.

Fukue et al. [1,15] investigated the cooling performance of this miniature piezoelectric ultrasonic micro-blower. They reported that this device can generate airflow at speeds of about $20 \mathrm{~m} / \mathrm{s}$, and it can be used for cooling electronics in narrow passages. They also performed CFD modeling of the response, assuming the micro-blower works like a continuous jet. However, this assumption was not verified directly.

In a number of earlier studies of this device, the primary focus was its bulk response, leaving the underlying mechanisms behind its performance unclear. Thus, we have examined the flow physics for this device using particle image velocimetry (PIV). We have conducted timeaveraged flow analysis in order to better understand the driving mechanisms that affect the heat transfer of free and impinging jets. We summarize the goals of the current study:

- $\quad$ Find the best operating conditions of the ultrasound micro-blower by measuring the diaphragm deflection.

- Examine the flow mechanisms of both a free and an impinging blower jet, particularly at the best operating frequency.

- Examine the heat transfer performance of the impinging micro-blower jet and compare to other synthetic jets and continuous jets.

- Calculate the coefficient of performance (COP) to find the best operating condition for this micro-blower.

First, we present experimental measurements of actuator deflection, which helps in the selection of the optimal frequency. Second, we present PIV measurements of the flow dynamics for free and impinging jets. Third, we present the corresponding heat transfer and coefficients of performance, and we discuss their implications.

\section{Experimental Methods}

\subsection{Mechanical Measurements}

Synthetic jets function through the motion of an oscillating driver, specifically a piezoelectric disk in this case. The mechanical response of this disk is critical to the flow response, since its 
amplitude directly relates to the cavity volume. This motion is dependent on the natural frequency of the disk, which is typically selected for optimal flow response. Of course, the disk mechanical motion is not the sole factor in the thermal performance, as the fluid dynamics inside and outside the cavity are critical. Hence, we use an experimental apparatus that measures the mechanical deflection as well as the convective heat transfer.

Figure 3 shows the test fixture, which is described in detail in previous publications $[2,10]$. The jet is placed on a traverse, which is used to fix its position to micron-scale accuracy. This actuator is driven by using a function generator, whose sinusoidal output is amplified with a separate instrument. For mechanical measurements, a laser vibrometer is aligned with the centerline of the actuator, and it measures the deflection to sub-micron accuracy. This device has an uncertainty of $\pm 3 \%$.

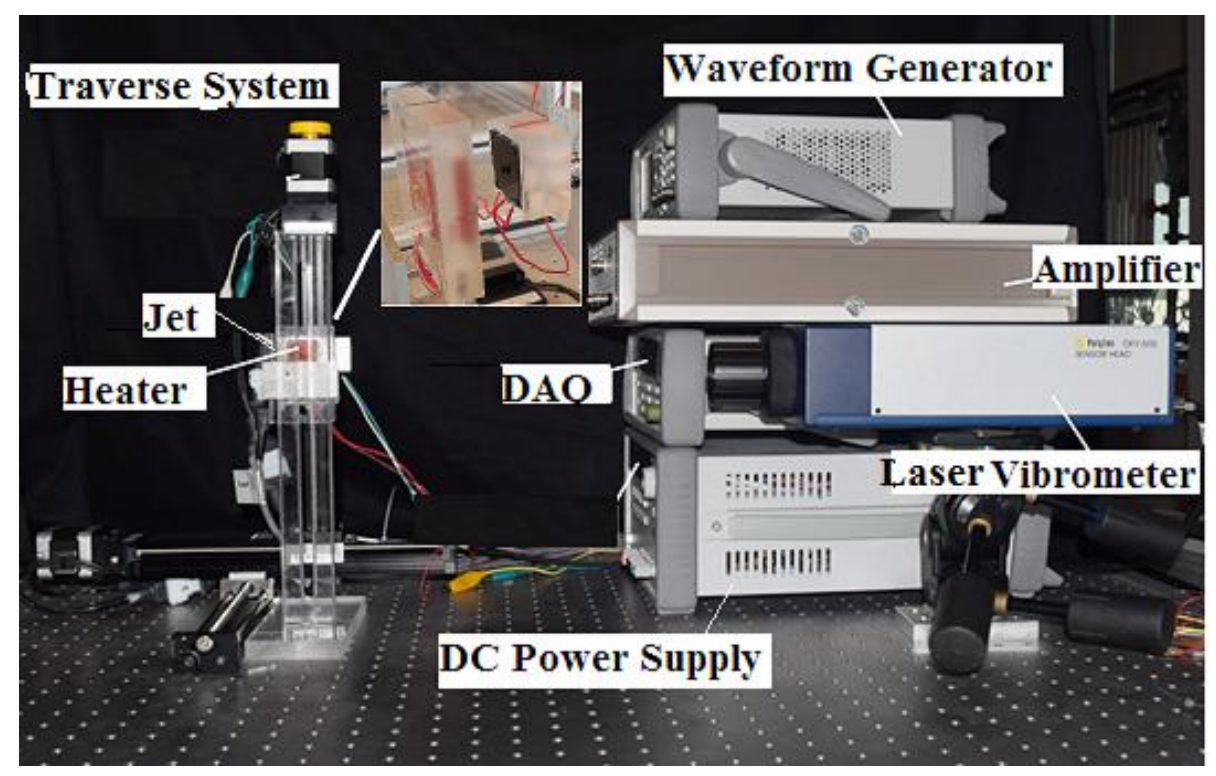

Figure 3. Image of the thermal and deflection measurement apparatus.

\subsection{Thermal Measurements}

The same test fixture is used for measuring the thermal response. The jet is placed normal to a square polyimide heater, 25.4-mm on a side. This device is arranged between a pair of copper plates, which help to produce a uniform surface temperature. A DC power supply operates the heater, whose resistive losses are dissipated via conduction, natural convection, and radiation. Since only forced convection from the front heater surface is needed for the analysis of the synthetic jet performance, other heat transfer modes are subtracted from the total heat transfer. 
We use a series of experimental and computational analyses to determine the conduction, natural convection, and radiation losses, using methods explained in detail earlier $[2,6,10]$. Conduction and natural convection losses are examined using a three-step process, initially developed by Garg et al. [6]. In step 1, the heater is operated outside of its Plexiglas test fixture, using only its wires for support. The micro-blower is initially inactive, so the heater dissipation is balanced by losses due to natural convection. This heat transfer is the same on the front and the back of the thin heater, so the natural convection losses may be evaluated for each side. In step 2, the heater is mounted in the Plexiglas substrate, and the power dissipation is measured at various heater temperatures. The natural convective loss from step 1 is subtracted from the total, leaving the conductive losses to the substrate, $Q_{\text {loss }}$. Computational simulations [10] verified the magnitude of these losses, agreeing to within 5\% of the experimental measurements. In step 3, the heater is operated with the micro-blower active, and the total heat transfer, $Q_{t o t}$, is measured. Then, the heat transfer from the front of the heater surface, $Q_{\text {surface }}$, is evaluated as:

$$
Q_{\text {surface }}=Q_{\text {tot }}-Q_{\text {loss }}
$$

Next, the radiative losses, $Q_{\text {radiation }}$, are calculated using the material emissivity and temperatures. These are subtracted from $Q_{\text {surface }}$ to determine the convective heat transfer, and the average convective heat transfer coefficient is calculated:

$$
h=\frac{Q_{\text {surface }}-Q_{\text {radiation }}}{A_{s}\left(T_{w}-T_{\text {amb }}\right)}
$$

Here, $A_{s}$ is the surface area of the heater. $T_{w}$ is the surface temperature of the heater, and $T_{a m b}$ is the temperature of the air in the enclosure. The surface temperature is determined by averaging the outputs of four thermocouples located behind the copper plate surface.

In each experiment, the heater temperature is set to a fixed value of $60^{\circ} \mathrm{C}$, as determined using the thermocouples behind the heater plate. An additional thermocouple measures the ambient temperature. The system is treated as steady once the temperature standard deviation is less than $0.1{ }^{\circ} \mathrm{C}$ over the last thirty readings, as sampled using a custom data acquisition software.

In further analysis, the key derived parameter is the Nusselt number, $N u=h D / k$, whose experimental uncertainty is determined using a standard propagation of uncertainties [16]. Here, $h$ is the average convective heat transfer coefficient given by Equation (2), $D$ is the actuator exit diameter, and $k$ is the air thermal conductivity. To evaluate the overall 
uncertainty, partial derivatives of $N u$ are taken with respect to each variable to determine sensitivity coefficients. Then, these coefficients are multiplied by the uncertainties in those individual variables, and the terms are root-sum-squared to find the overall uncertainty in $\mathrm{Nu}$. Of these variables, the major sources of uncertainty were due to the heater power and temperature measurements. The instrument uncertainties are $\pm 1 \%$ for heater voltage, $\pm 1 \%$ for heater current, and $\pm 0.4 \%$ for thermocouple temperature. The total heater power is simply the multiple of its voltage and current. When combined using the sensitivity coefficients, the overall uncertainty in $\mathrm{Nu}$ is estimated at approximately $\pm 6 \%$.

\subsection{Particle Image Velocimetry Measurements}

Subsequent to the mechanical and thermal studies, the full-field flow response was examined using PIV technique. Here, a separate fixture was used, as described elsewhere [12]. This is a rigid cubical tank, with approximately $60-\mathrm{cm}$ side length, as depicted in Figure 4 . The chamber has acrylic plates located on the top and side for camera visualization and an acrylic slot is used for the laser sheet. The micro-blower is rigidly mounted at a circular port in a side wall, and a Plexiglas plate is placed normal to it during impingement tests.

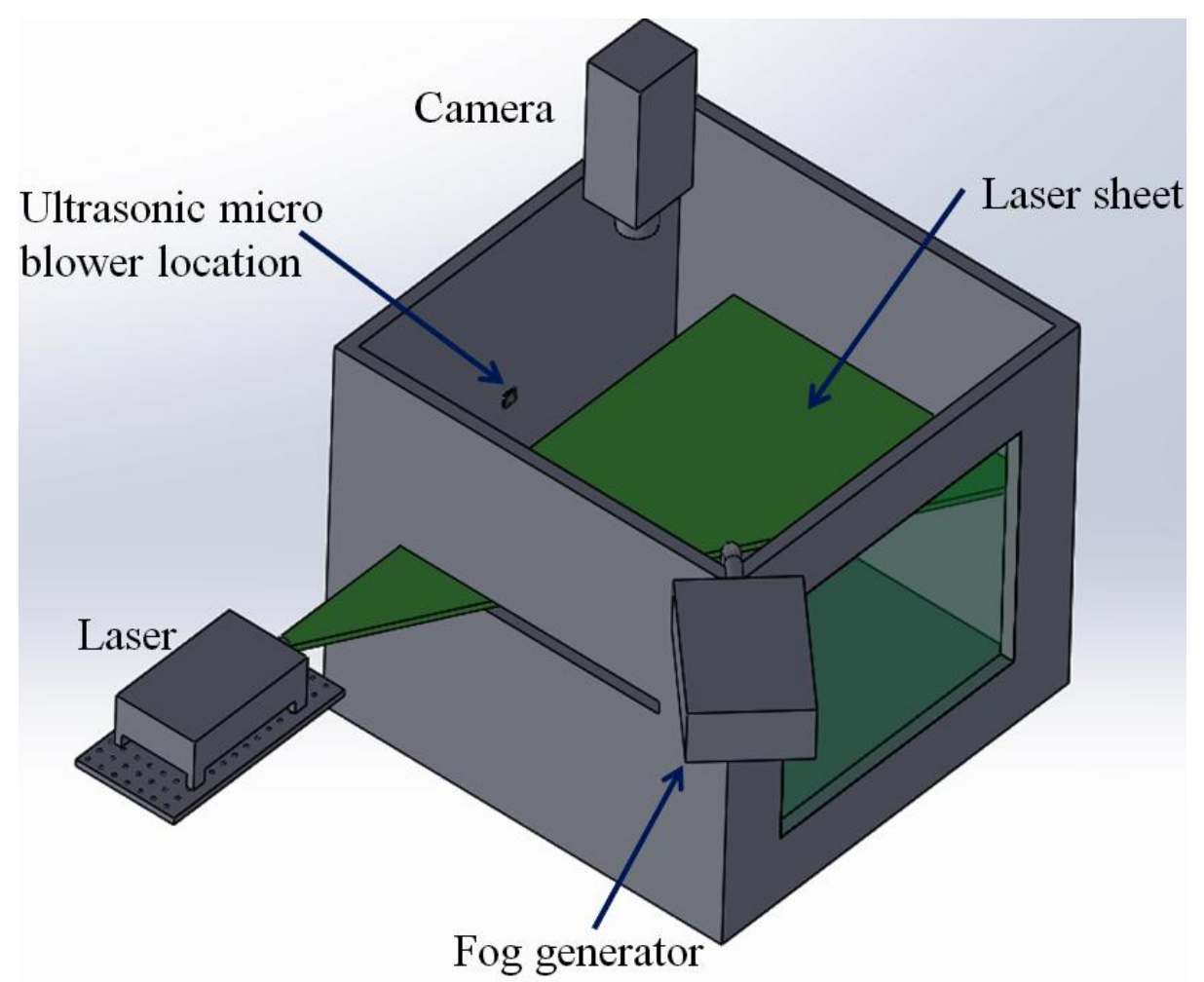

Figure 4. Schematic of the experimental apparatus, showing the cubical test section, microblower location, and the PIV system. 
The flow is seeded with micron-scale aqueous glycerol droplets, which are produced using a fog generator. These tracers are injected at the top of the test chamber prior to each test, and then a transparent lid is placed over the fixture to allow the system to settle. Then, a dual-head laser fires a pair of 50-mJ, 532-nm pulses at a delay of $10 \mu \mathrm{s}$ apart. Cylindrical and spherical lenses are used to concentrate the pulses into sheets, which are focused in a plane through the centerline of the actuator. The laser illuminates the droplets, and a 1.4-MP digital camera captures their locations from a position directly above the micro-blower. The laser and camera are synchronized externally, and the system is controlled with commercial PC software.

For each test condition, 500 image pairs are acquired. These images are analyzed with an open-source, a MATLAB based code [17]. This software splits the images into smaller regions, which are cross-correlated to determine the local tracer motion and corresponding velocity. The interrogation regions are iteratively decreased in size, resulting in high spatial resolution of approximately $1 \mathrm{~mm}$ by $1 \mathrm{~mm}$. From a propagation of uncertainty analysis [16], the estimated PIV uncertainty is approximately $\pm 4 \%$, primarily due to the processor resolution.

\section{Results and Discussion}

\subsection{Frequency Response}

Synthetic jets are strongly dependent on the operating frequency, with performance accentuated near their fundamental frequencies. Two frequencies usually stand out: a structural one due to the disk deflection, and a fluid one due to the Helmholtz cavity resonance. For this ultrasonic blower, the unique multi-orifice structure results in a more complex frequency response. The micro-blower has a Helmholtz resonant frequency around $21 \mathrm{kHz}$ when using the exhaust nozzle [10], and it has a resonance at $15.5 \mathrm{kHz}$ for its inner cavity alone [9]. In principle, the design could match the diaphragm resonant frequency and the cavity Helmholtz resonance for the best performance, but that might lead to noise at frequencies below the audible limit of $20 \mathrm{kHz}$. Thus, the micro-blower is designed to operate in a different mode. Usually, the first mode of the diaphragm is chosen for synthetic jet operation, but the micro-blower is operated at the third mode $[9,14]$, likely to aid acoustics.

To examine the mechanical response, the deflection of the jet was measured for a range of frequencies from $1 \mathrm{kHz}$ to $27 \mathrm{kHz}$. The samples had an interval of $100 \mathrm{~Hz}$ in order to capture 
the detailed deflection around the key modes of the actuator. Figure 5 displays the change of the deflection at the center of the diaphragm for the frequency range $23 \mathrm{kHz}<f<27 \mathrm{kHz}$. For the rest of the sample range, the deflection was below $2 \mu \mathrm{m}$. Here, the applied voltage is 20 $V_{p p}$ (peak-to-peak voltage). The maximum deflection obtained during the experimental study was approximately $13 \mu \mathrm{m}$ at $f=25 \mathrm{kHz}$. Hence, we set the device operating condition at this frequency, as it should have a high time-averaged jet velocity.

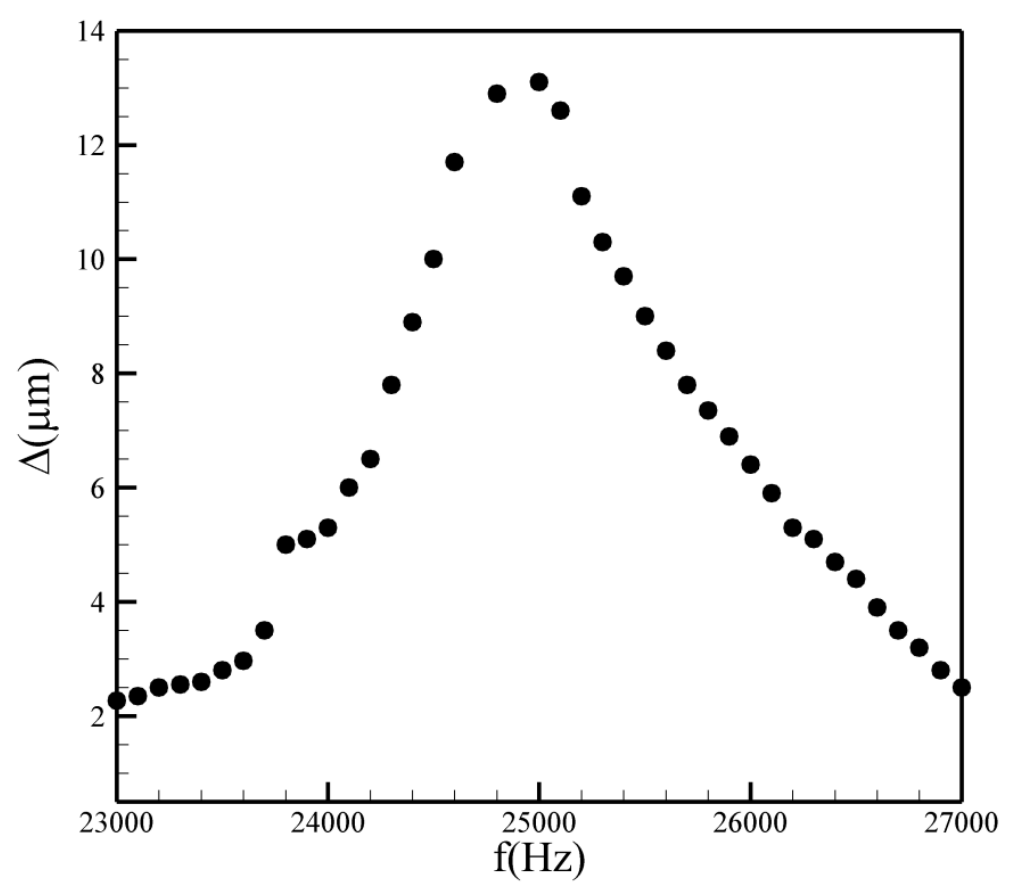

Figure 5. Variation of deflection at the center of the actuator diaphragm versus applied frequency at $20 V_{p p}$ (Voltage peak-to-peak).

\subsection{PIV Flow Visualization}

In order to understand the flow field associated with micro-blower impingement cooling, the flow behavior was investigated using PIV measurements. In this section, we first present the time-averaged results for a free synthetic jet. Then, we present the time-averaged results for an impinging blower jet. For each case, a total of 500 image pairs were acquired, and the resulting flow fields were ensemble-averaged. The PIV measurements examine a 2-D slice along the centerline of the jet. Figure 6 shows the schematic of the impinging jet, where $H$ is the jet-to-surface spacing. 


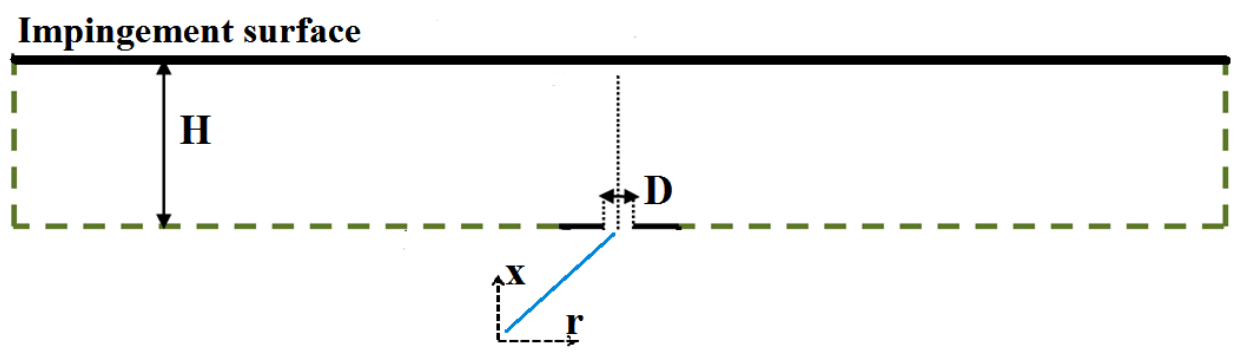

Figure 6. Geometry of the impinging jet.

Figure 7 shows the time-averaged velocity vectors and vorticity contours for an upwarddirected, free jet. Here, the jet operates at a Reynolds number of 1300 and a frequency of 25 $\mathrm{kHz}$. At this specific condition, the flow rate is approximately 0.94 LPM. The Reynolds number is defined as $R e=U_{\text {ave }} D / v$, where $U_{\text {ave }}$ is the average velocity at the nozzle, $D$ is the diameter of the exhaust nozzle, and $v$ is the air kinematic viscosity. Vorticity is normalized by the jet diameter and the average velocity. The jet spreads slightly as it travels upward, and it has a symmetric behavior along its centerline. Jet speeds are near zero with the first 5 diameters downstream for two reasons. First, while the primary inflow to this blower occurs away from the exhaust nozzle, there would be some return inflow in this region during the oscillating jet motion. Second, there were some artifacts due to reflection of laser light near the exit, which impacts the PIV cross-correlation accuracy.

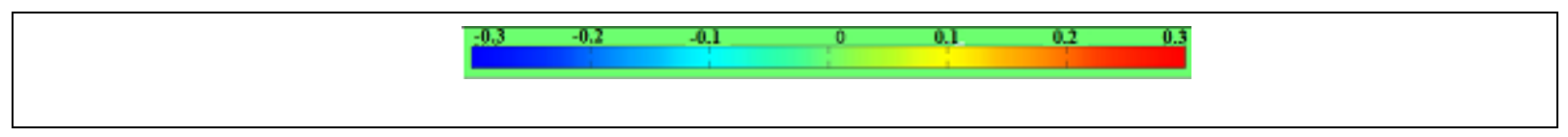




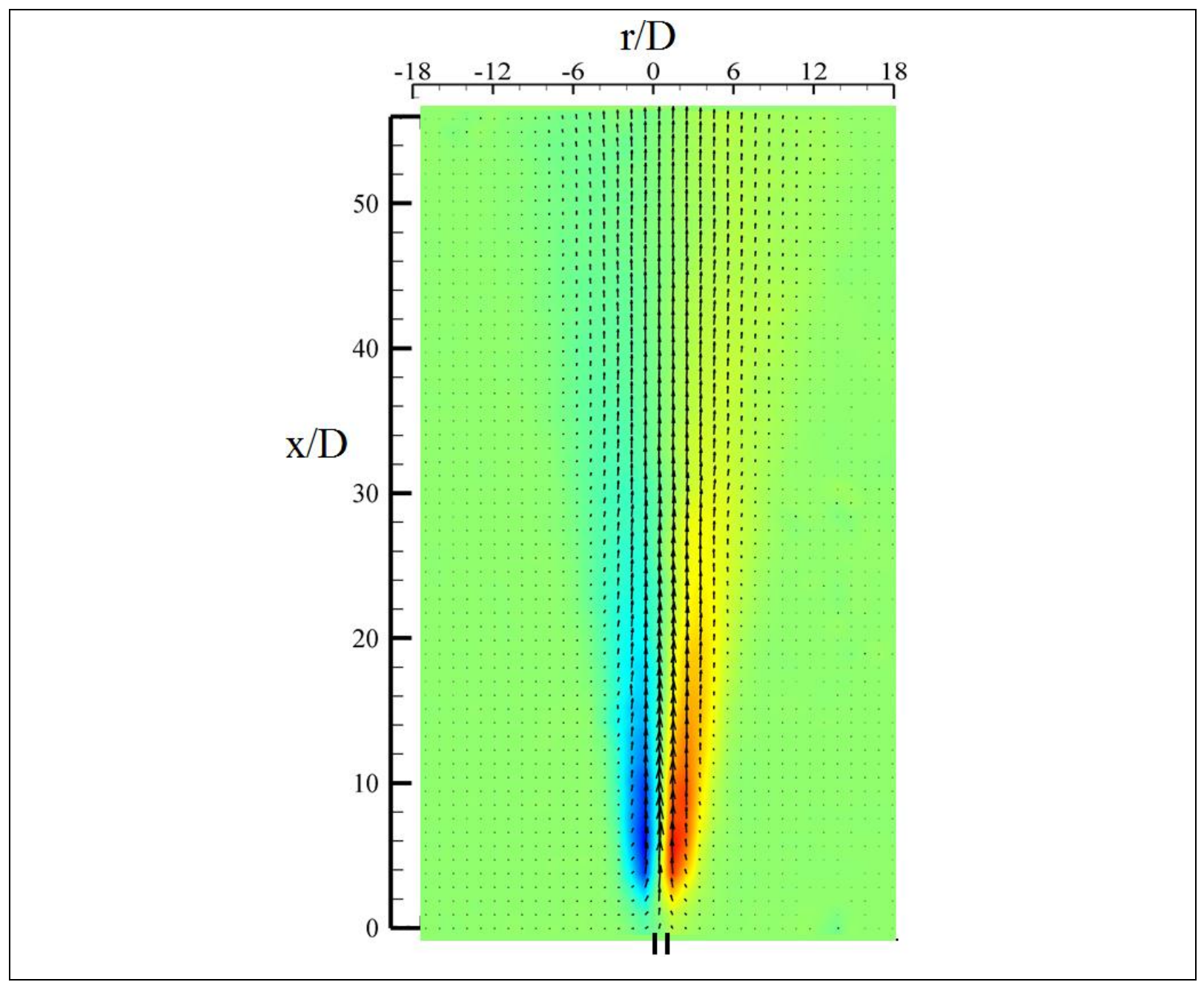

Figure 7. Velocity vectors and dimensionless vorticity $\left(\omega D / U_{\text {ave }}\right)$ for a free, ultrasonic microblower jet at $\operatorname{Re}=1300$.

Figure 8a shows the streamwise variation of normalized centerline velocity in the free jet at axial positions $x / D \geq 5$. The velocity shows continuous decay as jet moves upward. The jet spreads in the transverse direction, which results in a decrease of the centerline velocity. Ultimately, the decrease in the far field deviates only slightly from $1 / x$, as might be expected from a steady, axisymmetric jet. Figure $8 \mathrm{~b}$ shows the transverse distribution of streamwise velocity at different locations downstream from the nozzle. Between $x / D=5$ and 50 , the jet half-width increases by a factor of approximately 6 , and it appears to approach the expected Gaussian profile [18]. 

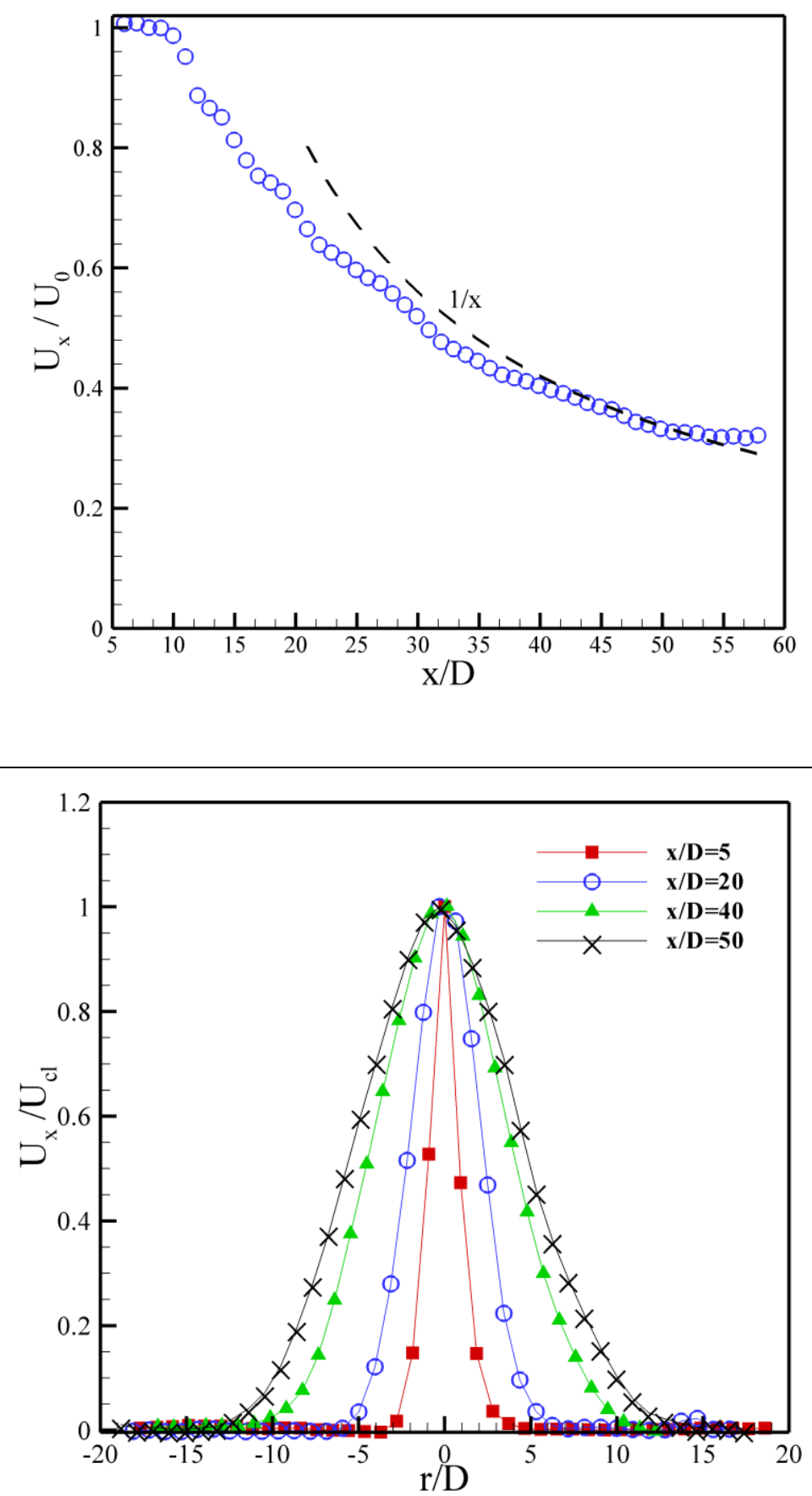

Figure 8. a) Streamwise variation of normalized centerline velocity in the free micro-blower jet b) Transverse distributions of streamwise velocity in the free jet. 
Figure 9a shows the time-averaged axial velocity profile at several axial positions. The jet axial velocity is normalized by the local centerline velocity, and the radial position is normalized by the half-width, $r_{1 / 2}$, of the profile. Here, the half-width is defined as the position where the axial velocity is half of the centerline speed. At the position closest to the exit, $x / D=5$, the profile shows a sharper peak at the centerline, while it has higher axial flow at the fringe of the jet than other locations. This differs from the response for a steady, developing jet, which has a flatter profile near the exit [19]. The profiles approach a Gaussian profile soon afterward, with little difference seen after $x / D=15$.

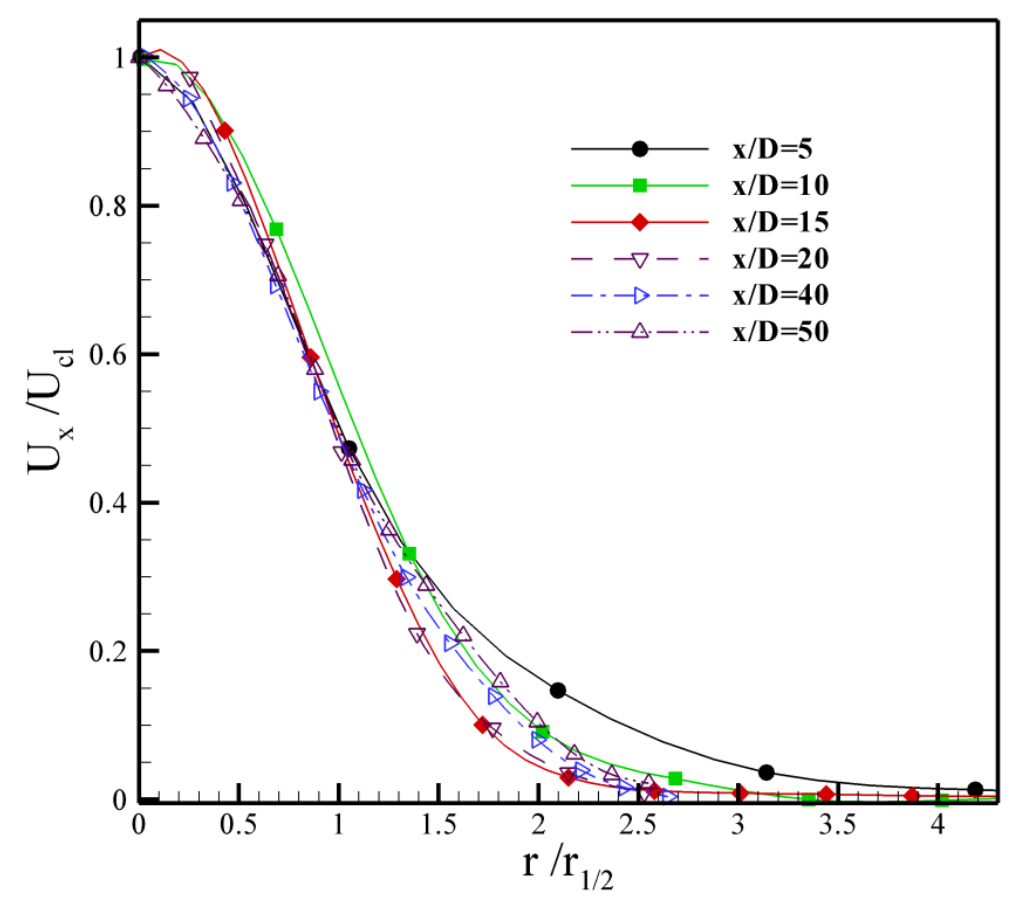

a) 


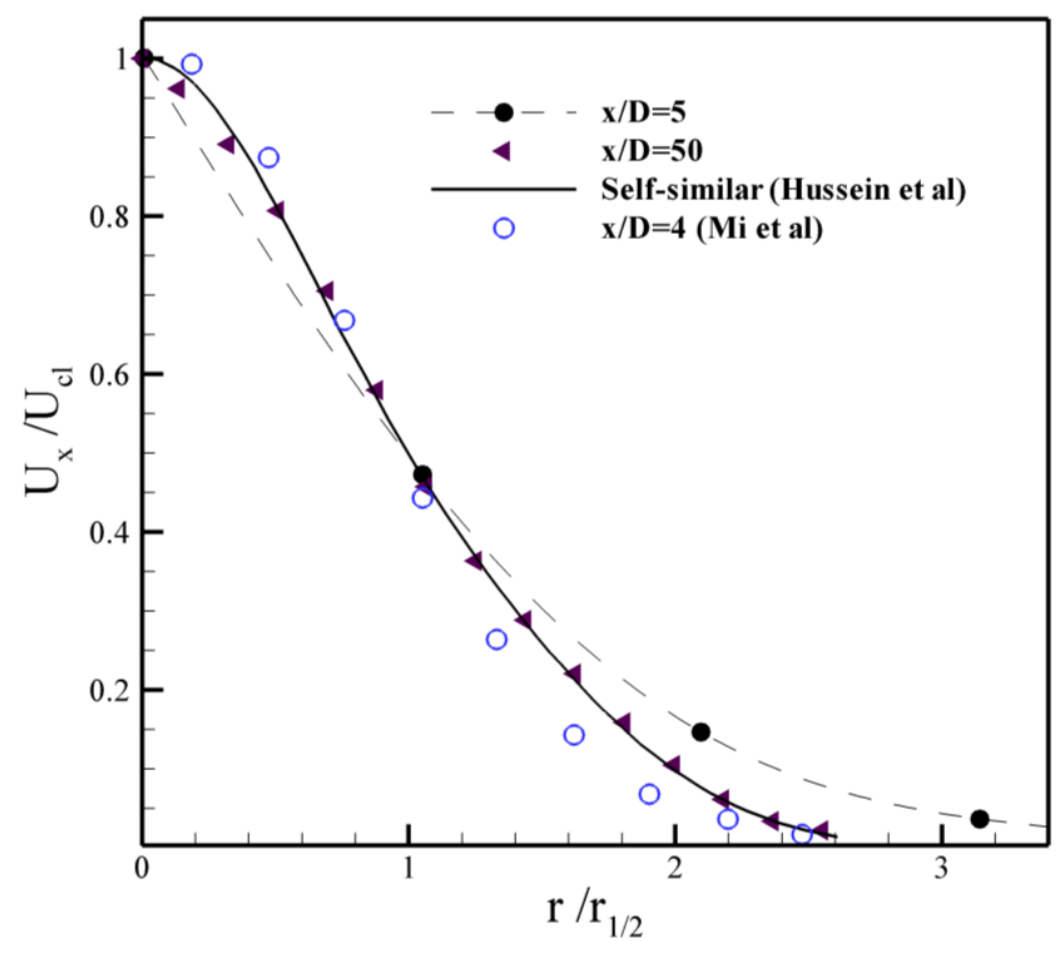

b)

Figure 9. Time-averaged, nondimensional axial velocity profiles a) Current study for different axial positions b) Comparison of near field and far field profiles to published data for a turbulent free jet $[18,19]$.

In the downstream region, $x / D>30$, a high-Reynolds number turbulent round jet should be self-similar, with a Gaussian profile [18]. As seen in Figure 9b, the micro-blower velocity profile shows excellent agreement with the self-similar turbulent round jet profile.

Interestingly, the micro-blower operates at a much lower $R e$ than the other cases shown (1300 versus 96000 [18] and 72000 [19]), yet the velocity profile is almost self-similar. This suggests that one can assume the far field $(x / D>40)$ of the jet behaves similar to a turbulent round jet. However, the near field of the micro-blower profile differs significantly from to near field of a steady turbulent jet [19], with a sharper peak and broader extent. Hence, a continuous jet CFD model [1] is a significant over-simplification compared to the actual ultrasonic micro-blower response.

Figure 10 shows the time-averaged vorticity contours for an impinging jet with a jet-to-wall spacing ratio of $H / D=40$. Since $D=1 \mathrm{~mm}$, this corresponds to a spacing of $H=40 \mathrm{~mm}$. Much like the free micro-blower jet (seen in Figure 7), the flow is symmetric, growing in 
width as it travels downstream. Unlike the free jet, there are two additional regions of vorticity next to the wall. These are due to the growing boundary layer as the wall jet travels along the wall. The peak vorticity magnitudes of this near wall region are less than the levels in the jet near the orifice.

Figure 11 shows the streamwise variation of the normalized, time-averaged centerline velocity for the impinging jet at $H / D=40$. The centerline velocity shows similar behavior to the free jet till $x / D=30$, but after that the flow is affected by the impingement wall. The velocity experiences a sharp decrease for $x / D>30$, and it reaches zero at the stagnation point. Figure 12 shows the transverse distributions of streamwise velocity for the impinging jet compared to the free jet at $x / D=35$. Here, the flow profiles are identical within the measurement uncertainty, except at radii far from the centerline. At these positions, the wall broadens the profile as it impinges.

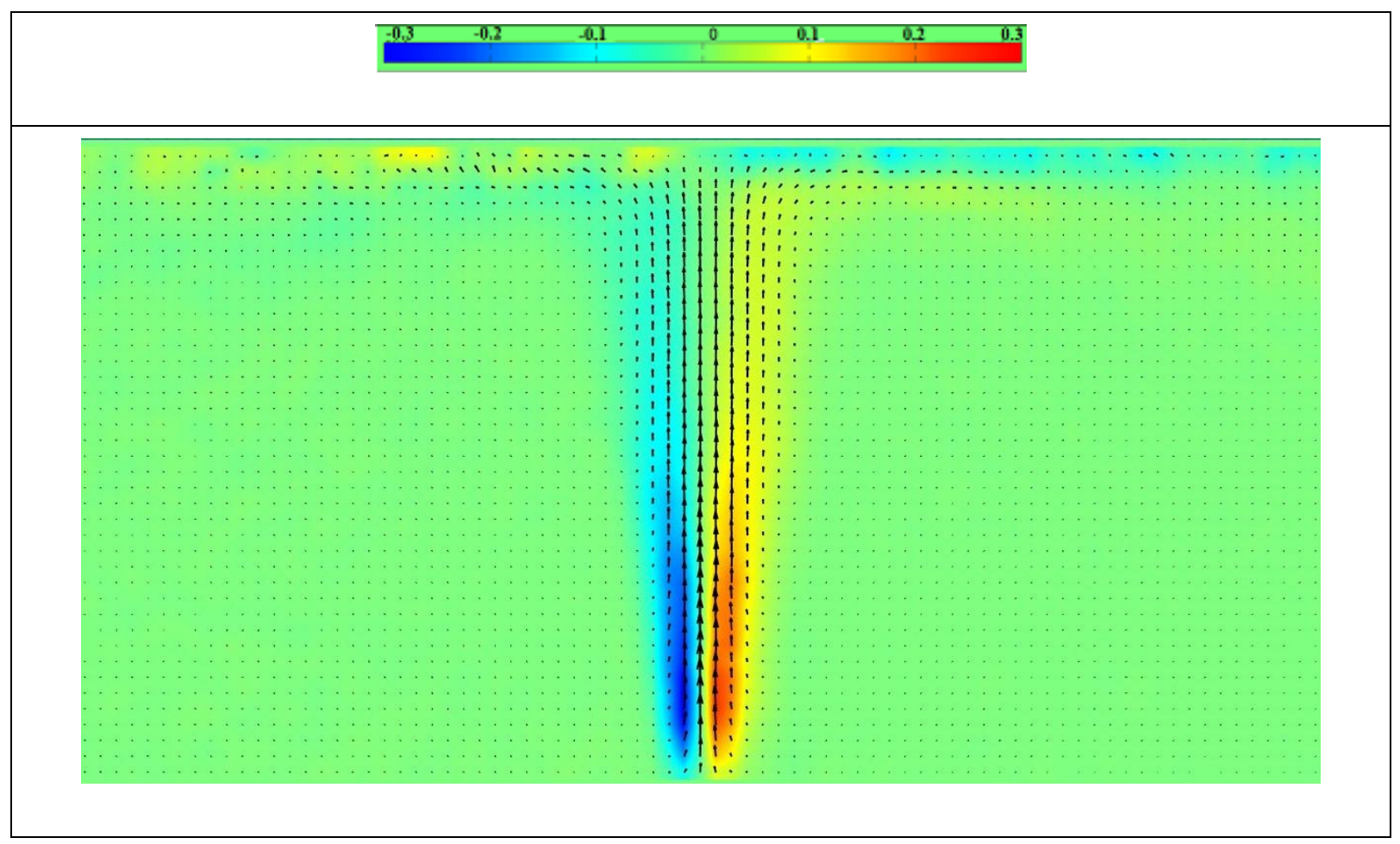

Figure 10 . Time-averaged velocity vectors and vorticity contours, $\omega D / U_{\text {ave }}$ for an impinging slot synthetic jet at $R e=1300$ at $H / D=40$. 


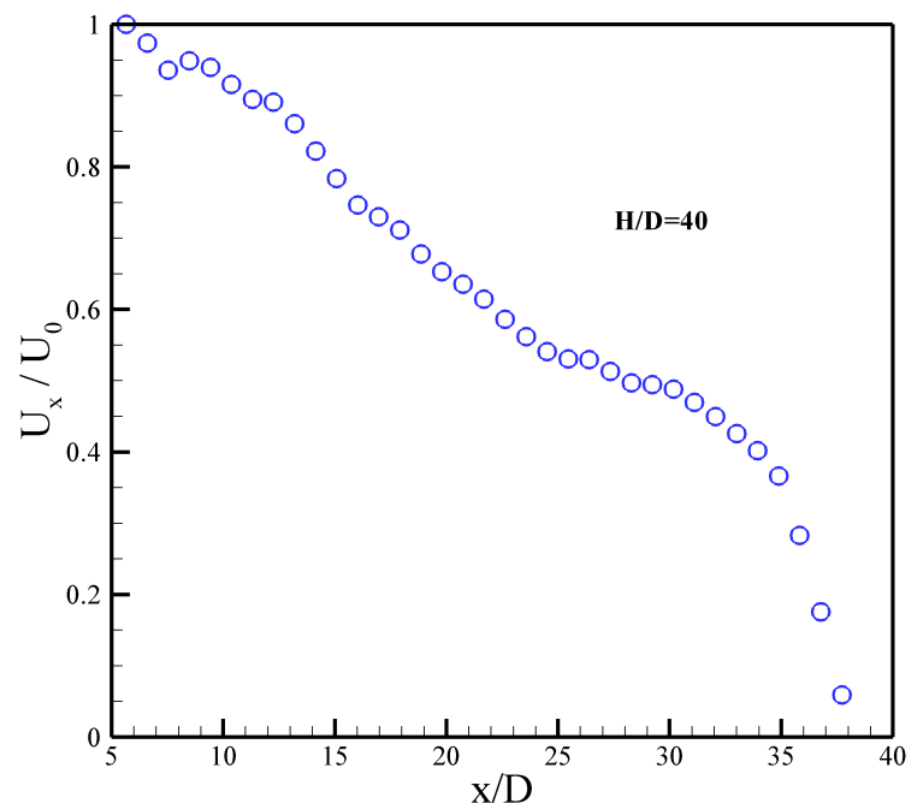

Figure 11. Streamwise variation of normalized, time-averaged centerline velocity for the impinging jet at $H / D=40$.

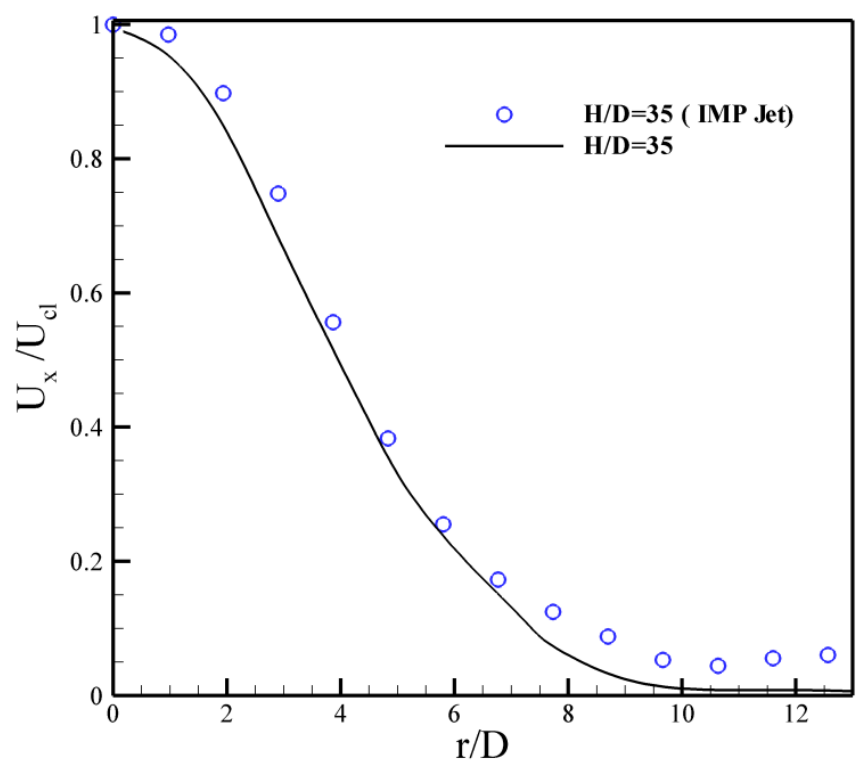

Figure 12. Transverse distribution of streamwise velocity in the impinging and free jets.

\subsection{Heat Transfer}


Figure 13 shows the experimental results for the average Nusselt number at different jet-tosurface spacings at $f=25 \mathrm{kHz}$. The average Nusselt number increases sharply at positions up to $H / D=10$, and then it shows a gradual rise till $H / D=15$. There is a fairly flat maximum region for $15 \leq H / D \leq 30$, followed by a gradual decay for $H / D>30$. The heat transfer increases more than three times when moving the jet from $H / D=2$ to $H / D=10$. This reveals that the jet performance is highly sensitive to the jet-to-surface spacing, and it decreases significantly for small jet-to-surface spacings. This sets a practical limit on the design and the flexibility in device placement in applications, especially for compact systems.

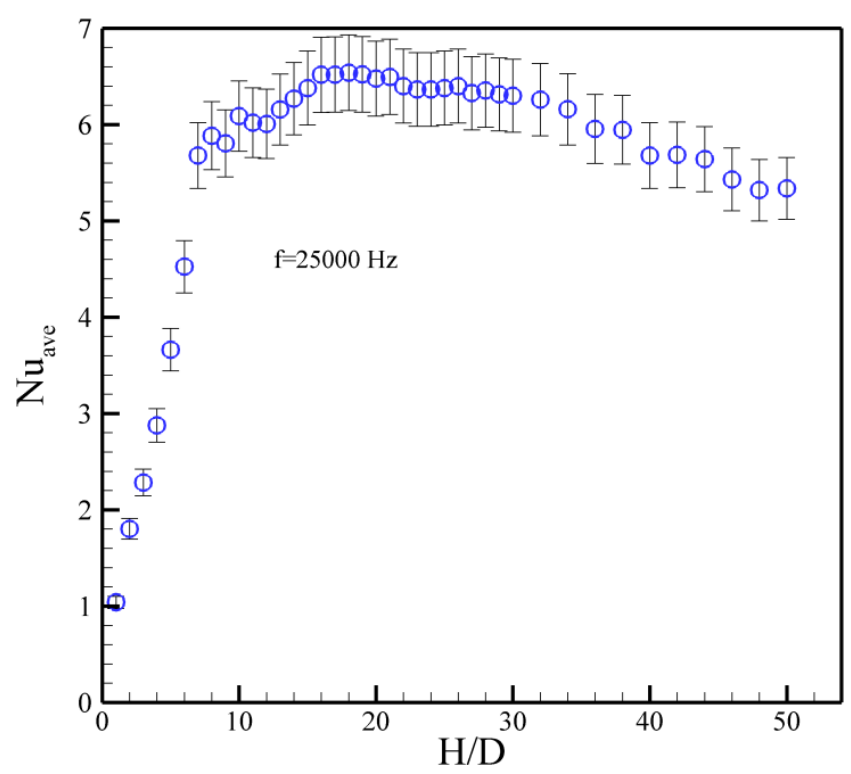

Figure 13. Variation of average Nusselt number with different jet-to-surface spacings at $f=25$ $\mathrm{kHz}$ and $R e=1300$.

An impinging synthetic jet typically has the maximum heat transfer performance at jet-tosurface spacings from $5 \leq H / D \leq 10$ [20, 21]. However, for continuous jets [22, 23], there is a monotonic decay of heat transfer with increasing jet-to-surface spacing at this Reynolds number. Much like the synthetic jet, the ultrasonic micro-blower has different heat transfer behavior within its near field of operation, although the peak response is further from the jet orifice. These differences suggest that the jet development and response is significantly altered in the near field. Hence, a continuous jet CFD model [1] will likely deviate from the ultrasonic micro-blower response. In the far field, the response will be more comparable, as partly revealed in the heat transfer correlation of Ikhlaq et al. [9]. Here, the micro-blower performance was not affected by jet frequency for $H / D>10$. 
Figure 14 shows the variation of average Nusselt number versus frequency at $H / D=10$. The heat transfer peaks at $f=25 \mathrm{kHz}$, which further suggests that the jet has its maximum flow rate here [9]. Off of this peak frequency, the heat transfer decreases by less than $10 \%$ between $24.5 \mathrm{kHz}$ and $25.5 \mathrm{kHz}$. However, the performance decreases rapidly below $24 \mathrm{kHz}$ and above $26 \mathrm{kHz}$. This degradation corresponds with the deflection measurements shown in Figure 5, which shows significant decreases in disk motion off of the resonant peak. Since the device flow rate is closely related to the maximum deflection, it is reasonable that the thermal performance would similarly decrease. Thus, while there is some flexibility with the operating condition near the peak, the blower effectiveness is strongly dependent on frequency.

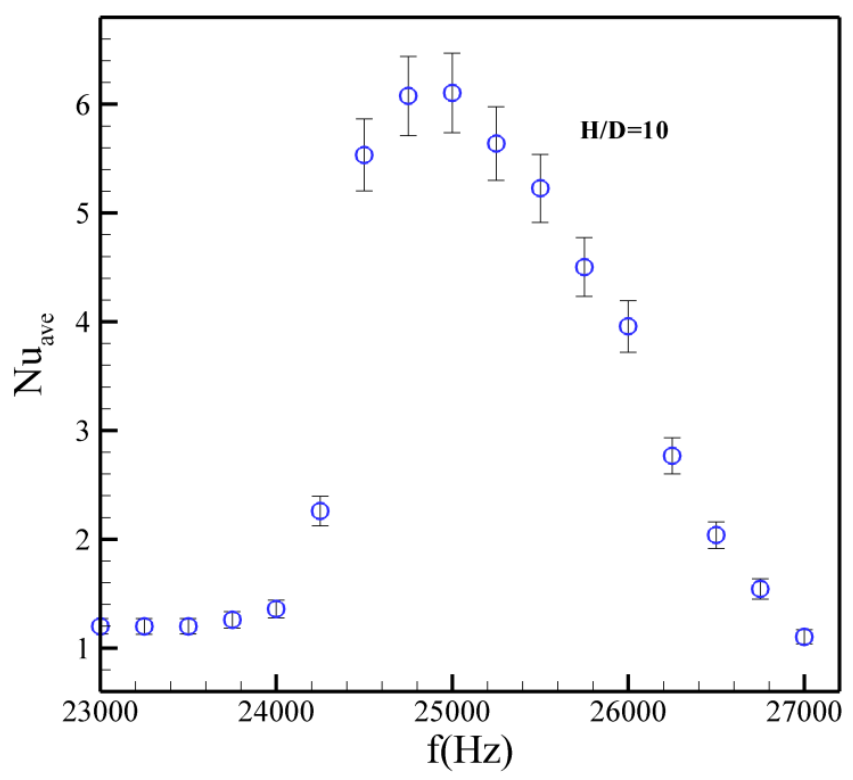

Figure 14. Variation of average Nu number with frequency for an impinging round jet at $H / D$ $=10$.

Finally, since the micro-blower requires power, the purported thermal benefit must be compared to this input. The natural metric here is the coefficient of performance (COP) [24], defined as the ratio between the convective heat transfer and the input power. Figure 15 shows the variation of $C O P$ at different frequencies for $H / D=10$. Here, the highest COP is 3 at $f=26 \mathrm{kHz}$, suggesting that the preferred operating condition may be off of the resonant peak. Thus, even though the heat transfer is higher at $f=25 \mathrm{kHz}$, the additional power consumption at this operating condition makes it less appealing. The COP at $25 \mathrm{kHz}$ is around 
2, which is much less than the values seen with slot synthetic jets, which have COP 14 [2]. Thus, while this micro-blower can greatly reduce noise, it has a significant performance penalty.

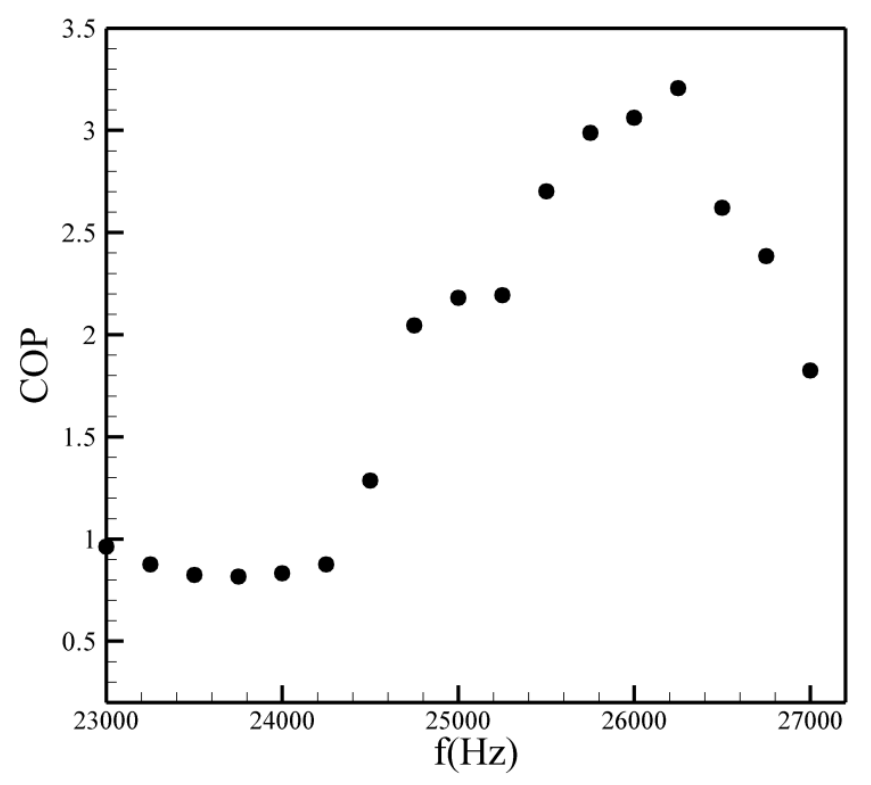

Figure 15. Variation of COP with frequency for a micro-blower jet at $H / D=10$.

\section{Summary and Conclusions}

We performed a series of experiments to examine the performance of an impinging ultrasonic micro-blower jet. Along with measurements of deflection and thermal performance, we used time-averaged PIV to study the flow physics and their effects on heat transfer. Specifically, the findings in this study can be summarized as:

The preferred operating frequency of the piezoelectric actuator occurs at an ultrasonic frequency of $25 \mathrm{kHz}$, meaning that this device can function with low noise.

The micro-blower axial velocity profile shows similar behavior to high Reynolds number turbulent free jets in the far field, including a self-similar profile. In the near field, it has significant deviation, showing a sharper peak than a steady jet. Both free and impinging flow fields differ from a steady jet in this region.

The average $\mathrm{Nu}$ number increases sharply up to $H / D=10$, and then it shows a gradual increase till $H / D=15$. This reveals that the jet performance is highly sensitive to the jet-to-surface spacing, particularly in the near field. 
The jet cooling performance is sensitive to the frequency, though there is a $1 \mathrm{kHz}$ wide band of similar thermal response about the peak.

The coefficient of performance at the best operating heat transfer condition is about 2 , which is less than the value of 14 seen for a slot synthetic jet [2]. Thus, while this micro-blower can greatly reduce noise, it has a performance penalty.

\section{Acknowledgments}

This work is partly supported by the Turkish National Science Foundation (TUBITAK) under the contract number 112M154 and the Istanbul Development Agency under Contract ISTKA-BIL26 and Contract ISTKA-TR-10/15/YNK/0029 .The authors would also like to thank the technical assistance of Chad Swanson and Troy Dunmire during the first author's research visit to Washington State University Vancouver.

\section{References}

[1] T. Fukue, K. Hirose, H. Terao, Cooling performance of impinging jet from piezoelectric micro blower mounted in narrow flow passage, 2015 Int. Conf. Electron. Packag. iMAPS All Asia Conf. (2015) 605-610. doi:10.1109/ICEPIAAC.2015.7111086.

[2] O. Ghaffari, M. Ikhlaq, M. Arik, An Experimental Study of Impinging Synthetic Jets for Heat Transfer Augmentation, Int. J. Air-Conditioning Refrig. 23 (2015) 1550024. doi:10.1142/S2010132515500248.

[3] E. Gutmark, E., Y. Yassour, and M. Wolfshtein, Acoustic Enhancement of Heat Transfer in Plane Channels, in: Proc. Seventh Int. Heat Transf. Conf. Munich, Ger. Sep. 6-10, 19825: pp. 441-445.

[4] Y. Yassour, J. Stricker, and M. Wolfshtein, Heat Transfer from a Pulsating Jet, in: Proc. Eighth Int. Conf. San Fr. CA, Aug. 17-22, Vol. 3, Pp. 1183-1186, 1986: pp. 1183-1186.

[5] A. L. Minichiello, J. G. Hartley, A. Glezer, and W. Z. Black, Thermal Management of Sealed Electronic Enclosures Using Synthetic Jet Technology, Adv. Electron. Packag. 19 (1997) 1809-1812.

[6] J. Garg, M. Arik, S. Weaver, T. Wetzel, S. Saddoughi, Meso Scale Pulsating Jets for Electronics Cooling, J. Electron. Packag. 127 (2005) 503. doi:10.1115/1.2065727. 
[7] Y. Utturkar, M. Arik, C. E. Seeley, M. Gursoy, An Experimental and Computational Heat Transfer Study of Pulsating Jets, J. Heat Transfer. 130 (2008) 062201. doi:10.1115/1.2891158.

[8] C. J. M. Lasance, R. M. Aarts, O. Ouweltjes, Synthetic jet cooling part II: Experimental results of an acoustic dipole cooler, Annu. IEEE Semicond. Therm. Meas. Manag. Symp. (2008) 26-31. doi:10.1109/STHERM.2008.4509361.

[9] M. Ikhlaq, O. Ghaffari, M. Arik, Predicting Heat Transfer for Low and High Frequency Central Orifice Synthetic Jets, IEEE Trans. Components .Packaging IEEE

Components, Packag. Manuf. Technol. (2016). doi:10.1109/TCPMT.2016.2523809.

[10] M. Ikhlaq, O. Ghaffari, M. Arik, Effect of Actuator Deflection on Heat Transfer for Low and High Frequency Synthetic Jets, in: IEEE Intersoc. Conf. Therm.

Thermomechanical Phenom. Electron. Syst., IEEE, Orlando, FL, USA, 2014.

[11] M. Arik, An investigation into feasibility of impingement heat transfer and acoustic abatement of meso scale synthetic jets, Appl. Therm. Eng. 27 (2007) 1483-1494. doi:10.1016/j.applthermaleng.2006.09.027.

[12] S. A. Solovitz, O. Ghaffari, M. Arik, Frequency-dependent flow response of a highspeed rectangular synthetic jet, Int. J. Heat Fluid Flow. (2016), submitted for publication.

[13] M. Jabbal, S. Kykkotis, Towards the Noise Reduction of Piezoelectrical-Driven Synthetic Jet Actuators, in: 32nd AIAA Appl. Aerodyn. Conf. June 2014, Atlanta, GA, 2014: pp. 1-21.

[14] K. Kurihara, United States Patent, Fujisaki et al. Patent N0.: (45) Date of Patent: US 8,596,998 B2 Dec. 3, 2013,” 2013, 2013.

[15] T. Fukue, Y. Matsuura, K. Hirose, H. Terao, Evaluation of cooling performance of a piezoelectric micro blower in narrow flow passage, 2014 Int. Conf. Electron. Packag. (2014) 69-73. doi:10.1109/ICEP.2014.6826663.

[16] R. J. Moffat, Describing the uncertainties in experimental results, Exp. Therm. Fluid Sci. 1 (1988) 3-17. doi:10.1016/0894-1777(88)90043-X.

[17] W. Thielicke, E.J. Stamhuis, PIVlab - Towards User-friendly, Affordable and Accurate Digital Particle Image Velocimetry in MATLAB, J. Open Res. Softw. 2 (2014). doi:10.5334/jors.bl. 
[18] H.J. Hussein, S.P. Capp, W.K. George, Velocity measurements in a high-Reynoldsnumber, momentum-conserving, axisymmetric, turbulent jet, J. Fluid Mech. 258 (2006) 31. doi:10.1017/S002211209400323X.

[19] J. Mi, P. Kalt, G. J. Nathan, C. Y. Wong, PIV measurements of a turbulent jet issuing from round sharp-edged plate, Exp. Fluids. 42 (2007) 625-637. doi:10.1007/s00348007-0271-9.

[20] A. Pavlova, M. Amitay, Electronic Cooling Using Synthetic Jet Impingement, J. Heat Transfer. 128 (2006) 897. doi:10.1115/1.2241889.

[21] O. Ghaffari, S. A. Solovitz, M. Arik, An Investigation into Flow and Heat Transfer for a Slot Impinging Synthetic Jet, Int. J. Heat Mass Transf. (2016), submitted for publication.

[22] N. Zuckerman, N. Lior, Jet Impingement Heat Transfer: Physics, Correlations, and Numerical Modeling, Advances in Heat Transfer, 39 (2006) 565-631. doi:10.1016/S0065-2717(06)39006-5.

[23] M. Y. Wen, K. Jang, An impingement cooling on a flat surface by using circular jet with longitudinal swirling strips, Int. J. Heat Mass Transf. 46 (2003) 4657-4667. doi:10.1016/S0017-9310(03)00302-8.

[24] S.A. Solovitz, M. Arik, System-Level Metrics for Thermal Management Technology, J. Therm. Sci. Eng. Appl. 3 (2011) 031009. doi:10.1115/1.4004486. 